Supplement of Biogeosciences, 15, 669-691, 2018

https://doi.org/10.5194/bg-15-669-2018-supplement

(C) Author(s) 2018. This work is distributed under

the Creative Commons Attribution 3.0 License.

(c) (1)

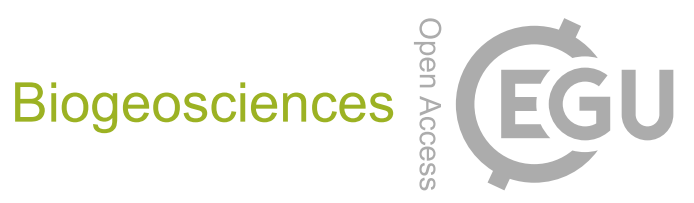

Supplement of

\title{
Hydro-ecological controls on dissolved carbon dynamics in groundwater and export to streams in a temperate pine forest
}

Loris Deirmendjian et al.

Correspondence to: Loris Deirmendjian (lorisdeir@gmail.com)

The copyright of individual parts of the supplement might differ from the CC BY 3.0 License. 
Supplementary Material

\begin{tabular}{|c|c|c|c|c|c|}
\hline \multirow[t]{2}{*}{ Period } & \multirow[t]{2}{*}{ Date } & \multicolumn{3}{|c|}{ Groundwater } & \multirow{2}{*}{$\begin{array}{c}\text { Surface water } \\
\text { First-order streams }\end{array}$} \\
\hline & & Piezometer Bilos & Piezometer 2 & Piezometer 3 & \\
\hline $\mathrm{HF}$ & $29 / 01 / 2014$ & & & & $\mathrm{X}$ \\
\hline $\mathrm{HF}$ & $12 / 02 / 2014$ & $\mathrm{X}$ & & & \\
\hline $\mathrm{HF}$ & $07 / 03 / 2014$ & & & & $\mathrm{X}$ \\
\hline $\mathrm{HF}$ & $17 / 03 / 2014$ & $\mathrm{X}$ & & & \\
\hline GS & $24 / 04 / 2014$ & & & & $\mathrm{X}$ \\
\hline GS & $16 / 05 / 2014$ & $\mathrm{X}$ & & & \\
\hline GS & $21 / 05 / 2014$ & & & & $\mathrm{X}$ \\
\hline GS & $25 / 06 / 2014$ & & & & $\mathrm{X}$ \\
\hline GS & $17 / 072014$ & $\mathrm{X}$ & & & $\mathrm{X}$ \\
\hline GS & $27 / 08 / 2014$ & $\mathrm{X}$ & $\mathrm{X}$ & & $\mathrm{X}$ \\
\hline LS & $24 / 09 / 2014$ & $\mathrm{X}$ & $\mathrm{X}$ & & $\mathrm{X}$ \\
\hline LS & $31 / 10 / 2014$ & $\mathrm{x}$ & $\mathrm{X}$ & & $\mathrm{x}$ \\
\hline EW & $21 / 11 / 2014$ & $\mathrm{X}$ & $\mathrm{X}$ & & $\mathrm{X}$ \\
\hline EW & $16 / 12 / 2014$ & $\mathrm{x}$ & $\mathrm{X}$ & & $\mathrm{X}$ \\
\hline EW & $27 / 01 / 2015$ & $\mathrm{X}$ & $\mathrm{X}$ & $\mathrm{X}$ & $\mathrm{X}$ \\
\hline $\mathrm{HF}$ & $04 / 03 / 2015$ & $\mathrm{X}$ & $\mathrm{X}$ & $\mathrm{x}$ & $\mathrm{x}$ \\
\hline GS & $10 / 04 / 2015$ & $\mathrm{x}$ & $\mathrm{X}$ & $\mathrm{X}$ & $\mathrm{X}$ \\
\hline GS & $07 / 05 / 2015$ & $\mathrm{x}$ & $\mathrm{x}$ & $\mathrm{x}$ & $\mathrm{x}$ \\
\hline GS & 03/06/2015 & $\mathrm{X}$ & $\mathrm{X}$ & $\mathrm{x}$ & $\mathrm{X}$ \\
\hline GS & 09/07/2015 & $\mathrm{X}$ & $\mathrm{X}$ & $\mathrm{X}$ & $\mathrm{X}$ \\
\hline
\end{tabular}

Table S1: Sampling dates of groundwater and surface waters. X correspond to a sampling of $\mathrm{pCO}_{2}$, total alkalinity and DOC. HF, GS, LS and EW represent respectively high flow (Jan. 2014-Mar. 2014 and Feb. 2015-Mar. 2015), growing 
season (Apr. 2014-Aug. 2014 and Apr. 2015-Aug. 2015), late summer (Sep. 2014-Oct. 2014 and Sep. 2015-Oct.

5 2015) and early winter (Nov. 2014-Jan. 2015 and Nov. 2015-Dec. 2015) periods. 


\begin{tabular}{|c|c|c|c|c|c|c|}
\hline \multirow[t]{2}{*}{ Period } & \multirow[t]{2}{*}{ Date } & \multirow{2}{*}{$\begin{array}{c}\text { DIC }_{\text {stock }} \text { and DOC } \text { stock } \\
\text { Piezometer Bilos }\end{array}$} & \multicolumn{3}{|c|}{ DIC $_{\text {export }}$ and DOC export } & \multirow{2}{*}{$\begin{array}{c}F_{\text {degass }} \\
\text { Streams }\end{array}$} \\
\hline & & & Piezometer Bilos & Piezometer 2 & Piezometer 3 & \\
\hline $\mathrm{HF}$ & $12 / 02 / 14$ and $17 / 03 / 14$ & $\mathrm{X}$ & $\mathrm{X}$ & & & $\mathrm{X}^{\mathrm{a}}$ \\
\hline GS & $17 / 03 / 14$ and $16 / 05 / 14$ & $\mathrm{X}$ & $\mathrm{X}$ & & & $\mathrm{X}^{\mathrm{b}}$ \\
\hline GS & $16 / 05 / 14$ and $17 / 07 / 14$ & $\mathrm{X}$ & $\mathrm{X}$ & & & $X^{c}$ \\
\hline GS & $17 / 07 / 14$ and $27 / 08 / 14$ & $\mathrm{X}$ & $\mathrm{X}$ & & & $\mathrm{X}$ \\
\hline LS & $27 / 08 / 14$ and $24 / 09 / 14$ & $\mathrm{X}$ & $\mathrm{X}$ & $\mathrm{X}$ & & $\mathrm{X}$ \\
\hline LS & $24 / 09 / 14$ and $31 / 10 / 14$ & $\mathrm{X}$ & $\mathrm{X}$ & $\mathrm{X}$ & & $\mathrm{X}$ \\
\hline EW & $31 / 10 / 14$ and $21 / 11 / 14$ & $\mathrm{X}$ & $\mathrm{X}$ & $\mathrm{X}$ & & $\mathrm{X}$ \\
\hline EW & $21 / 11 / 14$ and $16 / 12 / 14$ & $\mathrm{X}$ & $\mathrm{X}$ & $\mathrm{X}$ & & $\mathrm{X}$ \\
\hline EW & $16 / 12 / 14$ and $27 / 01 / 15$ & $\mathrm{x}$ & $\mathrm{X}$ & $\mathrm{X}$ & & $\mathrm{X}$ \\
\hline $\mathrm{HF}$ & $27 / 01 / 15$ and $04 / 03 / 15$ & $\mathrm{X}$ & $\mathrm{X}$ & $\mathrm{X}$ & $\mathrm{X}$ & $\mathrm{X}$ \\
\hline $\mathrm{HF}$ & $04 / 03 / 15$ and $10 / 04 / 15$ & $\mathrm{X}$ & $\mathrm{X}$ & $\mathrm{X}$ & $\mathrm{X}$ & $\mathrm{X}$ \\
\hline GS & $10 / 04 / 15$ and $07 / 05 / 15$ & $\mathrm{X}$ & $\mathrm{X}$ & $\mathrm{X}$ & $\mathrm{X}$ & $\mathrm{X}$ \\
\hline GS & $07 / 05 / 15$ and $03 / 06 / 15$ & $\mathrm{X}$ & $\mathrm{X}$ & $\mathrm{X}$ & $\mathrm{X}$ & $\mathrm{X}$ \\
\hline GS & $03 / 06 / 15$ and $09 / 07 / 15$ & $\mathrm{X}$ & $\mathrm{X}$ & $\mathrm{X}$ & $\mathrm{X}$ & $\mathrm{X}$ \\
\hline
\end{tabular}

Table S2: Periods of calculation for carbon stocks, carbon exports and carbon degassing. X corresponds to a calculation. $\mathrm{a}, \mathrm{b}, \mathrm{c}$ for these periods the day of sampling of surface waters do not correspond exactly to the day of sampling of groundwater (Tab. S1). Carbon stocks in groundwater can be calculated only for Bilos plot since we do not have data

10 about the total height of the permeable layer in the other plots. HF, GS, LS and EW represent respectively high flow (Jan. 2014-Mar. 2014 and Feb. 2015-Mar. 2015), growing season (Apr. 2014-Aug. 2014 and Apr. 2015-Aug. 2015), late summer (Sep. 2014-Oct. 2014 and Sep. 2015-Oct. 2015) and early winter (Nov. 2014-Jan. 2015 and Nov. 2015-Dec. 2015) periods. 
